\title{
Over-stimulation of insulin/IGF-1 signaling by Western diet may promote diseases of civilization: lessons learnt from Laron syndrome
}

\author{
Bodo C Melnik ${ }^{1 *}$, Swen Malte John ${ }^{1}$ and Gerd Schmitz ${ }^{2 \dagger}$
}

\begin{abstract}
The insulin/insulin-like growth factor-1 (IGF-1) pathway drives an evolutionarily conserved network that regulates lifespan and longevity. Individuals with Laron syndrome who carry mutations in the growth hormone receptor $(G H R)$ gene that lead to severe congenital IGF-1 deficiency with decreased insulin/IGF-1 signaling (IIS) exhibit reduced prevalence rates of acne, diabetes and cancer. Western diet with high intake of hyperglycemic carbohydrates and insulinotropic dairy over-stimulates IIS. The reduction of IIS in Laron subjects unmasks the potential role of persistent hyperactive IIS mediated by Western diet in the development of diseases of civilization and offers a rational perspective for dietary adjustments with less insulinotropic diets like the Paleolithic diet.
\end{abstract}

\section{Introduction}

Recently, Guevara-Aguirre et al reported on 99 Ecuadorian individuals with Laron syndrome due to growth hormone receptor $(G H R)$ deficiency and congenital insulin-like growth factor-1 (IGF-1) deficiency who did not develop type 2 diabetes (T2D) and were almost free of cancer, in contrast to their healthy relatives with normal insulin/IGF-1 signaling (IIS) [1]. A recent worldwide survey of Steuerman et al demonstrated that none of 230 individuals with Laron syndrome developed cancer [2]. Laron syndrome is a very informative experiment of nature and uncovers the link between low IIS and the related protection from diseases of civilization in contrast to exaggerated IIS induced by Western diet as shown in Figure 1.

Insulin/IGF-1/FoxO signaling in nonhuman organisms Over 20 years ago it was discovered that mutations in daf-2 and age- 1 double the lifespan in worms $[3,4]$. Daf2 encodes the only insulin/IGF-1 receptor expressed in worms and age-1 is the catalytic subunit of the downstream phosphoinositide 3-kinase (PI3K). Substantial evidence has been provided that IIS is an evolutionarily

\footnotetext{
* Correspondence: Melnik@t-online.de

† Contributed equally

'Department of Dermatology, Environmental Medicine and Health Theory,

University of Osnabrück, Sedanstrasse 115, D-49090 Osnabrück, Germany

Full list of author information is available at the end of the article
}

conserved pathway that regulates lifespan across many species like flies, worms, and mice [3-6]. Decreased IIS in nonhuman organisms has been associated with extended lifespan and protection against oxidative stress-mediated age-dependent damage [5,6]. IIS activates PI3K and Akt kinase. Akt-mediated phosphorylation activates the kinase mammalian target of rapamycin complex 1 (mTORC1) activating its effector S6 kinase 1 (S6K1) involved in the up-regulation of protein synthesis and cell proliferation. Furthermore, activated Akt stimulates specific phosphorylation of FoxO proteins in the nucleus leading to their extrusion into the cytoplasm [5]. FoxO transcription factors have emerged as a convergence point of IIS, nutrient availability and oxidative stress [6]. Increased expression of DAF-16, the ortholog of human FoxO proteins in the worm Caenorhabditis elegans, due to a mutation of the insulin/IGF-1 receptor daf-2 has been found to significantly increase the worm's lifespan [2,3]. Male and female heterozygous IGF-1 receptor knockout mice $I g f 1 r^{+/-}$live $16 \%$ and 33\% longer than wild-type males and females, respectively [7]. Thus, convincing experimental evidence obtained from invertebrates and nonhuman vertebrates taught us that down-regulated IIS is of critical importance for metabolic homeostasis, improved oxidative stress responses and longevity.

The pathogenesis of age-related diseases has been associated with an impaired capacity to counteract 


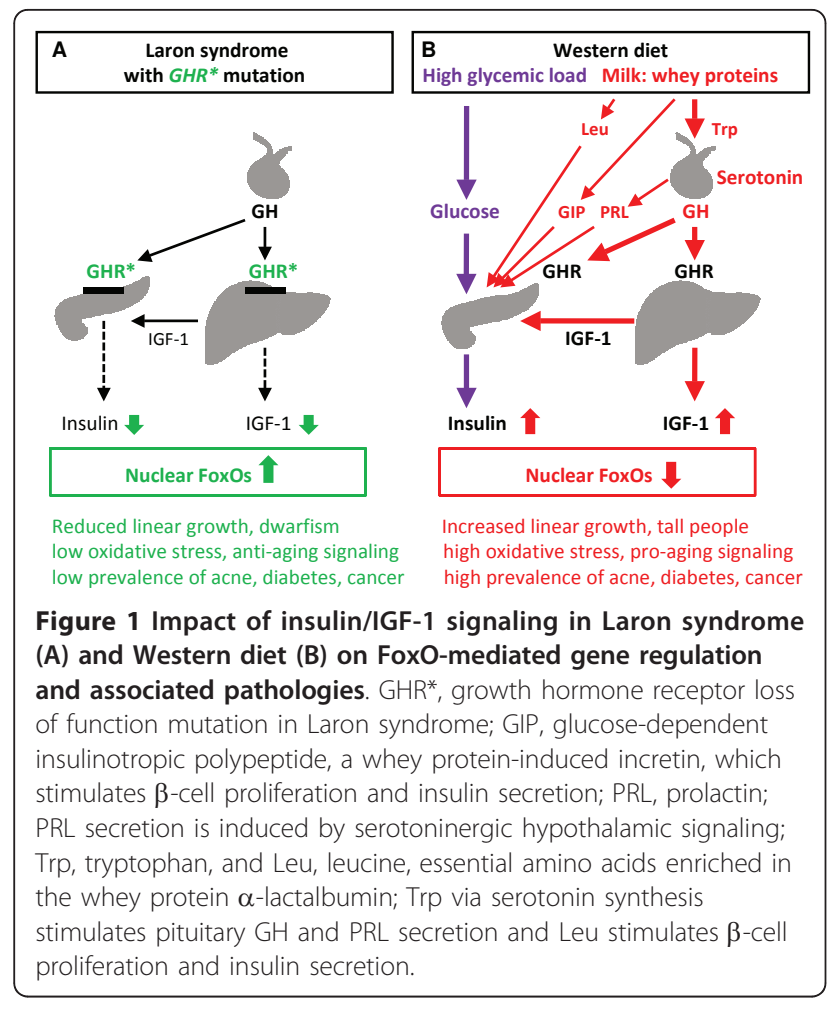

cellular damage induced by oxidative stress. In T2D some of the consequences of an oxidative environment are the development of insulin resistance, $\beta$-cell dysfunction, impaired glucose tolerance, and mitochondrial dysfunction [8]. Oxidative stress, implicated in the etiology of cancer, results from an imbalance in the production of reactive oxygen species (ROS) and the cell's own antioxidant protection. ROS deregulate the redox homeostasis and promote tumor formation by initiating an aberrant induction of signaling networks that cause tumorigenesis [9]. FoxO proteins are pivotal regulators of oxidative stress resistance and activate the expression of manganese superoxide dismutase and catalase [5]. Moreover, FoxO1 at the promoter level induces expression of Hmoxl (heme oxygenase 1) thereby decreasing mitochondrial respiration and ROS formation [5]. Thus, increased IIS with down-regulated nuclear FoxO levels impairs adequate elimination of ROS, a critical mechanism involved in the promotion of acne, T2D and cancer.

\section{Insulin/IGF-1/FoxO signaling and type 2 diabetes}

FoxO1 inhibits $\beta$-cell proliferation [10]. Nutritional alterations of $\beta$-cell FoxO1 transcriptional activity are predominantly mediated through glucose-stimulated insulin secretion and insulin receptor signaling. Recently, the concept of a "metabolic diapause" has been proposed for the changes induced by FoxO1 to protect $\beta$-cells against oxidative stress underpinning the concept of $\beta$-cell rest as a treatment goal in T2D [11]. Thus, FoxO1, the convergence point of IIS, orchestrates $\beta$-cell proliferation and apoptosis which both are increased in T2D [12].

\section{Insulin/IGF-1/FoxO signaling and cancer}

GH, IGF-1 and insulin have cancer-promoting actions and raised serum IGF-1 levels have been associated with increased risk of prostate, breast and colorectal cancers [13]. Steuerman et al conducted a large worldwide survey on the prevalence of cancer in patients with various causes of secondary congenital IGF-1 deficiency and confirmed that subjects with Laron syndrome with congenital IGF-1 deficiency seem protected from the development of cancer [2]. IIS regulates the nuclear distribution of FoxO proteins which are increasingly considered to represent unique cellular targets directed against human cancer in light of their pro-apoptotic effects and their ability to lead to cell cycle arrest $[5,14]$. FoxOs are involved in the control of angiogenesis, stem cell proliferation, cell adhesion, oxidative stress responses, as well as innate and acquired immunity. Permanently increased IIS with consecutive down-regulation of nuclear FoxO levels may thus promote the development of cancer [14].

\section{Insulin/IGF-1/FoxO signaling and acne}

Acne is epidemic in adolescents of Western life style and absent in populations consuming a Paleolithic diet excluding sugar, grains and dairy like the Kitava islanders who exhibit low basal insulin levels compared with age-matched Europeans [15]. Accumulating evidence supports the view that consumption of hyperglycemic carbohydrates and insulinotropic dairy are the driving force of the acne epidemic in Western countries [16]. IGF-1 is a strong stimulator of sebaceous lipogenesis and acne pathogenesis has been linked to elevated IIS with consecutive nuclear FoxO deficiency $[16,17]$. Moreover, IGF-1 stimulates androgen receptor signaling by increasing adrenal and gonadal androgen synthesis as well as androgen receptor transactivation by nuclear extrusion of the androgen receptor cosuppressor FoxO1 $[17,18]$. Thus, acne can be considered as a visible model disease of exaggerated IIS during puberty aggravated by Western diet, just the opposite of deficient IIS in untreated Laron subjects who do not develop acne [19]. On the other hand, seborrhea, acne, T2D and cancer are frequently observed in acromegaly with increased GH/IGF-1 signaling in comparison to healthy controls [16]. Intriguingly, an association between the prevalence of severe tetracycline-treated acne and increased risk of prostate cancer later in life has been observed [20]. 


\section{Western diet potentiates insulin/IGF-1 signaling}

Grain-based food, sugars and dairy products are the nutritional staples of Western diet. High glycemic load diets have been recognized as aggravating factors of acne, whereas a low glycemic load diet improved acne and decreased the bioavailability of free IGF-1 in plasma [21].

Milk and fermented dairy products containing whey proteins exhibit a high insulinemic index in comparison to their low carbohydrate content [22]. This phenomenon appears to be the secret of IIS of mammalian milk, an evolutionarily provided program for the promotion of neonatal growth [16]. Cow milk consumption in humans shifts the somatotropic axis to higher levels and significantly increases serum levels of GH and IGF-1 [23] (Figure 1B). Oral ingestion of the whey protein $\alpha$ lactalbumin has been shown to increase the somatotropic axis in healthy women. There is strong epidemiological evidence that dairy consumption significantly increases serum IGF-1 levels in humans [24]. This explains why high intake of milk increases linear growth [25]. In contrast, short stature is a characteristic feature of congenital IGF-1 deficiency in Laron syndrome. Thus, Western diet shifts the GH/IGF-1 axis to abnormally high levels, just in the opposite direction of low IIS observed in Laron syndrome [1,2] (Figure 1).

\section{Exaggerated insulin/IGF-1 signaling by Western diet and type 2 diabetes}

Insulin resistance and hyperinsulinemia are characteristic features of the metabolic syndrome. Extensive consumption of hyperglycemic food with increased glucosemediated signal transduction to pancreatic $\beta$-cells is a major factor of glucose/FoxO1-mediated $\beta$-cell proliferation and impaired $\beta$-cell oxidative stress responses [8-11].

Milk consumption after the weaning period maintains high levels of IIS persistently stimulating pancreatic $\beta$ cell proliferation [26]. Continued over-stimulation of pancreatic $\beta$-cells by whey protein-driven IIS after the post-weaning period may continuously diminish nuclear FoxO levels, thereby promoting oxidative stress damage of $\beta$-cells finally resulting in early onset of $\beta$-cell cellular senescence and apoptosis [26]. Indeed, increased proliferation and apoptosis of $\beta$-cells during lifetime are hallmarks of T2D [12].

\section{Exaggerated insulin/IGF-1 signaling of Western diet and cancer}

Increased IIS has been implicated to play an important role in most types of epithelial neoplasia [13,27]. Higher serum IGF-1 levels were associated with increased risk of cancer death in older community-dwelling men [28].
On the other hand, subjects with Laron syndrome exemplify that low IIS is associated with a low prevalence of cancer $[1,2]$. However, it should be mentioned that Laron subjects are not longer lived than normal subjects eating a Western diet and if not properly treated die of cardiovascular diseases.

\section{Conclusion and future perspectives}

Laron syndrome with decreased IIS is associated with a reduced prevalence of acne, T2D and cancer. In contrast, up-regulated IIS by Western diet appears to promote the development of chronic diseases of civilization. Paleolithic diet, which excludes hyperglycemic carbohydrates and insulinotropic dairy, has been successfully introduced for the prevention and treatment of acne, T2D and cardiovascular diseases [16,29]. Future efforts should be undertaken to lower the high insulinemic index of milk (I.I. 140) and other whey-based milk products to reach values of beef (I.I. 51) or cheese (I.I. 45) $[16,29]$. Furthermore, combinations of hyperglycemic carbohydrates and insulinotropic dairy with potentiating effects on IIS should be restricted.

Individuals with genetic single nucleotide polymorphisms (SNPs) resulting in hyperactive IIS may be at special risk for the development of age-related diseases, predominantly when their high intrinsic IIS is superposed by exaggerated IIS of Western diet. Intriguingly, genetic variations with reduced IIS due to SNPs of interacting components of the IIS cascades (GH1, IGF1, IGF1R, IRS1, FoxO1A, FoxO3A) and have been associated with increased longevity [30,31]. Thus, future research should consider the impact of interacting intrinsic genetic as well as extrinsic dietary factors involved in the regulation of IIS.

The access to higher amounts of insulinotropic and IGF-1-raising foods (sugar, grains and dairy) occurred about 10,000 years ago during the Neolithic Revolution and was further augmented by the Industrial Revolution. However, the human genome may not have adapted to this "recent switch" to higher IIS driven by Western diet. According to mitochondrial DNA data, modern humans with nearly similar genomic structure lived roughly 200,000 years ago and consumed a less insulinotropic Paleolithic diet. In this regards, it has been proposed to re-adapt our nutrition to the beneficial characteristics of our pre-agricultural diets [32]. The time point introducing a well-balanced Paleolithic diet may be a special issue of concern as proper IIS is important for the function of the reproductive and central nervous system. In adulthood however, dietary restrictions decreasing IIS may reduce the risk of age-associated pathology like proteotoxicity as recently demonstrated in a mouse model of Alzheimer disease [33]. 


\section{Abbreviations}

Age-1: catalytic subunit of PI3K of C. elegans; Akt: Akt kinase (protein kinase B); C. elegans: Caenorhabditis elegans; DAF-2: Insulin/IGF-1 receptor of C. elegans; DAF-16: dauer form 16 (FoxO ortholog in the worm); FoxO: forkhead box transcription factor class $\mathrm{O}$; $\mathrm{GH}$ : growth hormone; GHR: growth hormone receptor; G.I.: glycemic index; GIP: glucose-dependent insulinotropic polypeptide (gastric inhibitory polypeptide); I.I.: insulinemic index; IIS: insulin/IGF-1 signaling; IGF-1: insulin-like growth factor-1; IGF1R: insulin-like growth factor-1 receptor; IR: insulin receptor; IRS: insulin receptor substrate; PI3K: phosphoinositide-3 kinase; Leu: leucine; mTORC1; mammalian target of rapamycin complex 1; ROS: reactive oxygen species; S6K1: S6 kinase 1; T2D: type 2 diabetes; Trp: tryptophan.

\section{Author details}

'Department of Dermatology, Environmental Medicine and Health Theory, University of Osnabrück, Sedanstrasse 115, D-49090 Osnabrück, Germany. ${ }^{2}$ Institute for Clinical Chemistry and Laboratory Medicine, University Clinic of Regensburg, Franz-Josef-Strauss-Allee 11, D-93053 Regensburg, Germany.

\section{Authors' contributions}

BCM and GS researched data, contributed to discussion and conclusion. SMJ contributed to conclusion and reviewed/edited manuscript. All authors read and approved the final manuscript.

\section{Competing interests}

The authors declare that they have no competing interests.

Received: 27 March 2011 Accepted: 24 June 2011

Published: 24 June 2011

\section{References}

1. Guevara-Aguirre J, Balasubramanian P, Guevara-Aguirre M, Wei M, Madia F, Cheng CW, Wei M, Hwang D, Martin-Montalvo A, Saavedra J, Ingles S, de Cabo R, Cohen P, Longo VD: Growth hormone receptor deficiency is associated with a major reduction in pro-aging signaling, cancer, and diabetes in humans. Sci Transl Med 2011, 3:1-9.

2. Steuerman R, Shevah O, Laron Z: Congenital IGF-I deficiency tends to confer protection against post-natal development of malignancies. Eur J Endocrinol 2011, 164:485-489.

3. Kenyon C, Chang J, Gensch E, Rudner A, Tabtiang R: A C. elegans mutant that lives twice as long as wild type. Nature 1993, 366:461-464.

4. Friedman DB, Johnson TE: A mutation in the age- 1 gene in Caenorhabditis elegans lengthens life and reduces hermaphrodite fertility. Genetics 1988, 118:75-86.

5. Cheng Z, White MF: Targeting forkhead box 01 from the concept to metabolic diseases: lessons from mouse models. Antioxid Redox Signal 2011, 14:649-661.

6. Salih DAM, Brunet $A$ : FoxO transcription factors in the maintenance of cellular homeostasis during aging. Curr Opin Cell Biol 2008, 20:126-136.

7. Holzenberger M, Dupont J, Ducos B, Leneuve P, Géloen A, Even PC, Cervera P, Le Bouc Y: IGF-1 receptor regulates lifespan and resistance to oxidative stress in mice. Nature 2003, 421:182-187.

8. Rains $J$, Jain SK: Oxidative stress, insulin signaling, and diabetes. Free Radic Biol Med 2011, 50:567-575.

9. Acharya A, Das I, Chandhok D, Saha T: Redox regulation in cancer: a double-edged sword with therapeutic potential. Oxid Med Cell Longev 2010, 3:23-34.

10. Buteau J, Accili D: Regulation of pancreatic $\beta$-cell function by the forkhead protein FoxO1. Diabetes Obesity Metab 2007, 9(Suppl 2):140-146.

11. Buteau J, Shlien A, Foisy S, Accili D: Metabolic diapause in pancreatic $\beta$-cells expressing a gain-of-function mutant of the forkhead protein Foxo1. J Biol Chem 2007, 282:287-293.

12. Rhodes CJ: Type 2 diabetes - a matter of $\beta$-cell life and death? Science 2005, 307:380-384.

13. Clayton PE, Banerjee I, Murray PG, Renehan AG: Growth hormone, the insulin- like growth factor axis, insulin and cancer risk. Nat Rev Endocrinol 2011, 7:11-24.

14. Maiese K, Chong ZZ, Shang YC, Hou J: Clever cancer strategies with FoxO transcription factors. Cell Cycle 2008, 7:3829-3839.
15. Cordain L, Lindeberg S, Hurtado M, Hill K, Eaton SB, Brand-Miller J: Acne vulgaris. A disease of Western civilization. Arch Dermatol 2002, 138:1584-1590.

16. Melnik BC: Evidence for acne-promoting effects of milk and other insulinotropic dairy products. Nestle Nutr Workshop Ser Pediatr Program 2011, 67:131-145.

17. Smith TM, Gilliland K, Clawson GA, Thiboutot D: IGF-1 induces SREBP-1 expression and lipogenesis in SEB-1 sebocytes via activation of the phospho-inositide 3-kinase/Akt pathway. J Invest Dermatol 2008, 128:1286-1293.

18. Ma Q, Fu W, Li P, Nicosia SV, Jenster G, Zhang X, Bai W: FoxO1 mediates PTEN suppression of androgen receptor $\mathrm{N}$ - and C-terminal interactions and coactivator re-cruitment. Mol Endocrinol 2009, 23:213-225.

19. Ben-Amitai D, Laron Z: Effect of insulin-like growth factor-1 deficiency or administration on the occurrence of acne. J Eur Acad Dermatol Venereol 2010.

20. Sutcliffe $S$, Giovannucci $E$, Isaacs WB, Willett WC, Platz EA: Acne and risk of prostate cancer. Int J Cancer 2007, 121:2688-2692.

21. Smith RN, Mann NJ, Braue A, Mäkeläinen H, Varigos GA: The effect of a high- protein, low glycemic-load diet versus a conventional, high glycemic-load diet on biochemical parameters associated with acne vulgaris: a randomized, investigator-masked, controlled trial. J Am Acad Dermatol 2007, 57:247-256.

22. Holt S, Brand Miller J, Petocz P: An insulin index of foods: the insulin demand generated by $1000-\mathrm{kJ}$ portions of common foods. Am J Clin Nutr 1997, 66:1264-1267.

23. Rich-Edwards JW, Ganmaa D, Pollak MN, Nakamoto ER, Kleinman K, Tserendolgor U, Willett WC, Frazier AL: Milk consumption and the prepubertal somatotropic axis. Nutr J 2007, 6:28.

24. Crowe FL, Key TJ, Allen NE, Appleby PN, Roddam A, Overvad H, Grønbaek H, Tjønneland A, Halkjaer J, Dossus L, Boeing H, Kröger J, Trichopoulou A, Dilis V, Trichopoulos D, Boutron-Ruault MC, De Lauzon B, Clavel-Chapelon F, Palli D, Berrino F, Panico S, Tumino R, Sacerdote C, Bueno-de-Mesquita HB, Vrieling A, van Gils CH, Peeters PH, Gram IT, Skeie $G$, Lund $E$, et al: The association between diet and serum concentrations of IGF-I, IGFBP-1, IGFBP-2, and IGFBP-3 in the European Prospective Investigation into Cancer and Nutrition. Cancer Epidemiol Biomarkers Prev 2009, 18:1333-1340.

25. Hoppe C, Molgaard C, Michaelsen KF: Cow's milk and linear growth in industrialized and developing countries. Annu Rev Nutr 2006, 26:131-173.

26. Melnik BC: Milk signalling in the pathogenesis of type 2 diabetes. Med Hypotheses 2011, 76:553-559.

27. Pollak M: Insulin and insulin-like growth factor signalling in neoplasia. Nat Rev Cancer 2008, 8:915-928.

28. Major JM, Laughlin GA, Kritz-Silverstein D, Wingard DL, Barrett-Connor E: Insulin-like growth factor-I and cancer mortality in older men. J Clin Endocriol Metab 2010, 95:1054-1059.

29. Frassetto LA, Schloetter M, Mietus-Snyder M, Morris RC, Sebastian A: Metabolic and physiologic improvements from consuming a paleolithic, hunter-gatherer type diet. Eur J Clin Nutr 2009, 63:947-955.

30. Bonafe $\mathrm{M}$, Olivieri F: Genetic polymorphism in long-lived people: cues for the presence of an insulin/IGF-pathway-dependent network affecting human longevity. Mol Cell Endocrinol 2009, 299:118-123.

31. Lunetta KL, D'Agostino RB, Karasik D, Benjamin EJ, Guo CY, Govindaraju R, Kiel DP, Kelly-Hayes M, Massaro JM, Pencina MJ, Seshadri S, Murabito JM: Genetic correlates of longevity and selected age-related phenotypes: a genome-wide association study in the Framingham Study. BMC Med Genet 2007, 8(Suppl 1):13.

32. Carrera-Bastos P, Fontes-Villalba M, O'Keefe JH, Lindeberg S, Cordain L: The western diet and lifestyle and diseases of civilization. Res Rep Clin Cardiol 2011, 2:15-35.

33. Cohen E, Paulsson JF, Blinder P, Burstyn-Cohen T, Du D, Estepa G, Adame A, Pham HM, Holzenberger M, Kelly JW, Masliah E, Dillin A: Reduced IGF-1 signaling delays age-associated proteotoxicity in mice. Cell 2009, 139:1157-1169.

doi:10.1186/1743-7075-8-41

Cite this article as: Melnik et al:: Over-stimulation of insulin/IGF-1 signaling by Western diet may promote diseases of civilization: lessons learnt from Laron syndrome. Nutrition \& Metabolism 2011 8:41. 